ISSN 0001-6002/2001/43/3/119-127

Acta Médica Costarricense,(C2001

Colegio de Médicos y Cirujanos

\title{
Resistencia bacteriana a antibióticos en el Hospital San Juan de Dios, 1995-1999
}

\author{
Ricardo Boza-Cordero, ${ }^{1}$ Edith Barrantes-Valverde ${ }^{2}$
}

Justificación: La resistencia a antibióticos por parte de bacterias patógenas es un grave problema mundial. Su control ha sido difícil pero deben hacerse todos los esfuerzos por realizarlo. Conocer la epidemiología en las diferentes regiones y países se constituye en parte importante de este control. Este estudio se llevó a cabo con el fin de analizar el comportamiento de la resistencia en el hospital San Juan de Dios, centro de atención nacional de adultos de aproximadamente 700 camas.

Materiales y métodos: El estudio se basó en los datos obtenidos en los años 1995-1999 ya que en 1995 se inició el empleo de equipo automatizado VITEK ${ }^{\circledR}$ en este hospital. Se analizaron 2817 aislamientos de cocos Gram positivos (CGP) y 7626 de bacilos Gram negativos (BGN) obtenidos de todos los sitios anatómicos y fluidos corporales. Por ser un estudio retrospectivo, no se pudo definir cuáles aislamientos provenían de infecciones intra o extrahospitalarias.

Resultados: Staphylococcus aureus fue el CGP más frecuentemente aislado, mientras que el BGN más frecuente fue Escherichia coli. En más de $90 \%$ de los estafilococos aislados se demostró resistencia a la penicilina. La resistencia a la oxacilina en S.aureus se incrementó de 35\% en 1995 a 52\% en 1999 mientras que en los estafilococos coagulasa negativa, pasó de 70 a $77 \%$ en el mismo periodo. La resistencia a la cefalotina en S.aureus pasó de 35 a $50 \%$ mientras que en los estafilococos coagulasa negativa pasó de 65 a $76 \%$. La resistencia a la clindamicina en ambos grupos bacterianos se mantuvo relativamente estable. No se demostró resistencia a la vancomicina.

La mayoría de los enterococos aislados correspondieron a E. faecalis, $75 \%$ de ellos fueron sensibles a la penicilina y $50 \%$ no mostraron sinergismo de la gentamicina con los antibióticos que actúan sobre la pared. No se encontraron enterococos vancomicina resistentes. En las enterobacterias se observó un aumento importante de la resistencia a cefalosporinas de tercera generación. E. coli mostró un aumento de la resistencia a ceftazidima de 10\% en 1995 a 35\% en 1999, mientras que Klebsiella pmeumoniae pasó de $0 \%$ a 52\% para el mismo antibiótico en ese periodo. Con respecto a cefotaxima, mientras que en E.coli se incrementó la resistencia de 5 a $12 \%$, en $K$. pneumoniae se modificó de 5 a $24 \%$. Esto podría deberse a la presencia de B-lactamasas de espectro ampliado. La sensibilidad a ciprofloxacina en $E$. coli al igual que en $K$. pneumoniae se mantuvo estable. En cuanto a amicacina, E.coli pasó de 0\% de resistencia en 1995 a 15\% en 1999 y K. pneumoniae de 20 a 32\%. En Enterobacter cloacae se demostró aumento importante de la resistencia a cefalosporinas de tercera generación y a amicacina no así a la ciprofloxacina. En todas las enterobacterias se mantuvo una baja resistencia al imipenem.

En cuanto a BGN no fermentadores, se demostró en Pseudomonas aeruginosa un aumento de la resistencia a la mayoría de los antibióticos con excepción del imipenem y ceftazidima. No obstante Acinetobacter calcoaceticus presentó un incremento importante de la resistencia a amicacina (de 20 a 72\%), a cefalosporinas de tercera generación (de 0 a $61 \%$ ) y ciprofloxacina (57\%), manteniendo alta sensibilidad sólo a imipenem (95\%).

Descriptores: resistencia a antibióticos, antibióticos, infecciones bacterianas, resistencia a beta lactámicos, resistencia a vancomicina, enterococos, enterobacterias, resistencia en neumococos, $\beta$-lactamasas de espectro ampliado.

Recibido: 23 de abril de 2001.

Aceptado: 23 de agosto de 2001.

\footnotetext{
Abreviaturas: CIM, concentración inhibitoria mínima; CGP, cocos Gram positivos; BGN, bacilos Gram negativos; Amp, ampicilina; Cefa, cefalotina; Stx, sulfatrimetoprin

Servicio de Infectología, Hospital San Juan de Dios

2 Laboratorio de Bacteriología, Hospital San Juan de Dios
}

Correspondencia: Ricardo Boza Cordero. Servicio de Infectología, Hospital San Juan de Dios. San José, Costa Rica. Correo electrónico: ricardob49@hotmail.com
El uso de agentes antimicrobianos desde hace más de 50 años ha disminuido la morbi-mortalidad por diversas enfermedades infecciosas. ${ }^{1,2}$ No obstante, el empleo de estos medicamentos conlleva al menos dos costos: el económico, ya que generalmente son productos caros y el biológico, porque su utilización se ha asociado a la aparición de resistencia a ellos por parte de las bacterias. ${ }^{1}$ Esta resistencia se observó desde los primeros años de su empleo, principalmente por parte de los estafilococos contra las penicilinas. ${ }^{1}$ Actualmente, se 
conocen la mayoría de los mecanismos genéticos y bioquímicos de la resistencia bacteriana, ${ }^{2}$ lo que ha mejorado la comprensión de este fenómeno, sin embargo, estamos aún lejos de poder controlarlo eficientemente, lo que produce enormes costos económicos.

Se ha establecido que el empleo extenso de estos medicamentos, tanto en humanos como en animales, es un hecho necesario pero no suficiente para explicar el incremento de la resistencia bacteriana. Además de la presión selectiva que se ejerce en el ambiente microbiano, el estado inmunitario del hospedero, la presencia de cuerpos extraños, los microambientes bacterianos y factores propios de las bacterias involucradas, tienen un papel importante en el desarrollo de la resistencia. ${ }^{1-4}$

Para un control adecuado de este problema, es fundamental conocer su comportamiento epidemiológico a escala mundial, regional y local ${ }^{3,5-8}$ y en este sentido, se han desarrollado esfuerzos por conocer el comportamiento del fenómeno en diversas regiones del mundo, ${ }^{5-8}$ lo que nos ha posibilitado obtener una visión más amplia y objetiva. Sin embargo, en nuestro medio sólo existen datos aislados, lo que implica la necesidad de realizar estudios sistemáticos y en gran escala con el fin de comprender nuestra realidad.

En el Laboratorio de Bacteriología del Hospital San Juan de Dios, desde 1995 se emplea un equipo automatizado para el aislamiento e identificación de bacterias así como para la realización de las pruebas de sensibilidad a los antibióticos, lo que ha facilitado el estudio de este problema.

El objetivo del presente estudio es describir los resultados obtenidos en este hospital entre 1995 y 1999 en cuanto a la sensibilidad a varios antibióticos de los grupos bacterianos de cocos Gram positivos (CGP) y bacilos Gram negativos (BGN) más frecuentemente aislados de diversos sitios y comparar estos datos con los descritos en la literatura mundial, con el fin de poder desarrollar políticas tanto para el uso racional de antimicrobianos como para enfrentar el problema del incremento de la resistencia bacteriana a estos medicamentos.

\section{Materiales y Métodos}

Se revisaron los archivos del Laboratorio de Bacteriología del Hospital San Juan de Dios desde enero de 1995 hasta diciembre de 1999. No se incluyó el año 1996 por no contar con datos completos para todos los meses.

Se analizaron las bacterias aisladas de todos los sitios anatómicos y fluidos corporales de pacientes ingresados en el hospital o que acudieron a la consulta externa.

Se estudiaron por separado los resultados obtenidos con los cocos Gram positivos (Staphylococcus aureus, Staphylococcus epidermidis, Streptococcus pneumoniae y Enterococcus $s p$ ) y con bacilos Gram negativos (Escherichia coli, Klebsiella pneumoniae, Enterobacter cloacae, Pseudomonas aeruginosa y Acinetobacter calcoaceticus).
Se escogieron las especies bacterianas más frecuentemente aisladas en el laboratorio de bacteriología del Hospital San Juan de Dios y en las que se han demostrado más problemas de resistencia en la literatura mundial.

La identificación de los microorganismos se realizó utilizando el sistema automatizado VITEK ${ }^{\circledR}$ (bio Merieux, Inc.), el cual se basa en el uso de tarjetas de identificación con 30 diferentes pruebas bioquímicas, las que generan un reporte en 2 a 18 horas, con resultados que enlistan 1 ó 2 microorganismos con sus respectivos porcentajes de probabilidad.

Las pruebas de sensibilidad a los antibióticos se realizaron por el mismo sistema por el método de concentración inhibitoria mínima (CIM), en concentraciones equivalentes en eficacia al método estándar, utilizando diferentes antibióticos según el microorganismo evaluado.

Con respecto a Acinetobacter baumannii se mantuvo el nombre de la especie que aparece en la tarjeta original (Acinetobacter calcoaceticus).

En cuanto a Streptococcus pneumoniae se realizó la prueba de difusión a la oxacilina con disco de 1 ug. En el caso de que la zona de inhibición fuese $<19 \mathrm{~mm}$ se procedió a efectuar el E-test. Se clasificó la cepa como sensible si la CIM a la penicilina fue $<0.06 \mathrm{ug} / \mathrm{ml}$, intermedia si fue $<1 \mathrm{ug} / \mathrm{ml} \mathrm{y}$ resistente si resultó $>2 \mathrm{ug} / \mathrm{ml}$.

\section{Resultados}

Se estudiaron un total de 2817 aislamientos de CGP y de 7626 de BGN. Los principales antibióticos utilizados en el hospital son los correspondientes al cuadro básico de la Caja Costarricense de Seguro Social, a saber penicilina G, ampicilina, cefalexina, cefalotina, sulfa-trimetoprin, eritromicina, tetraciclina, oxacilina, metronidazol, clindamicina, ciprofloxacina, cefotaxima, ceftazidima, vancomicina e imipenem; estos cinco últimos son de uso restringido para especialistas.

Por ser un estudio retrospectivo, no fue posible definir si estas infecciones eran de origen intra o extrahospitalario, pero el $85 \%$ de las muestras fueron tomadas en pacientes internados. Cerca del $80 \%$ de los aislamientos provenientes de la consulta externa fueron de pacientes con sepsis urinaria. $\mathrm{El}$ análisis de una muestra de estos últimos, realizado en el año 2000, no mostró diferencias significativas en cuanto al comportamiento de la resistencia a antimicrobianos expuesta en el presente estudio. (Barrantes E, datos no publicados).

La distribución de los aislamientos se anota en las Figuras 1 y 2 .

1. Cocos Gram positivos: La sensibilidad a los estafilococos se anota en el Cuadro 1. La resistencia a oxacilina (meticilina) en $S$. aureus aumentó en el periodo estudiado de 35 a $52 \%$, pero la sensibilidad a la cefalotina y a la clindamicina se mantuvo estable. 
Se demostró una alta resistencia a la penicilina, cefalotina y oxacilina en Staphylococcus epidermidis (Cuadro 1).

No se encontró ningún aislamiento de estafilococos resistente a la vancomicina.

Fueron analizadas 61 aislamientos de $S$ pneumoniae de sangre o esputo. Sólo en una se logró demostrar sensibilidad intermedia a la penicilina lo que significa $98 \%$ de sensibilidad a este medicamento.

En 483 aislamientos de Enterococcus faecalis, se demostró sensibilidad a la penicilina en $85 \%$ de ellos pero sólo en el $50 \%$ de los mismos se observó sinergismo de la gentamicina (syn500 ó CIM $<500 \mathrm{ug} / \mathrm{ml}$ ) con los antibióticos que actúan sobre la pared bacteriana (penicilina, ampicilina, vancomicina). No se identificó resistencia a la vancomicina en ninguno de ellos.

Por ser muy escasos los aislamientos de Enterococcus faecium, no se obtuvieron datos adecuados en cuanto a su patrón de sensibilidad.

2. Bacilos Gram negativos: Con respecto a los BGN (Cuadros 2, 3 y 4) se encontró una disminución de la sensibilidad a las cefalosporinas de tercera generación (ceftazidima y cefotaxima) y a la ciprofloxacina de parte de la mayoría de las bacterias. Asimismo, a pesar de que las bacterias no fermentadoras Pseudomonas aeruginosa y Acinetobacter calcoaceticus continúan manteniendo altos porcentajes de sensibilidad hacia el imipenem, desde 1995 se ha observado un aumento de la resistencia del $5 \%$ al $12 \%$ para la primera y de 0 a $5 \%$ para la segunda.

\section{Figura 1}

Resistencia a antibióticos en Cocos Grampositivos Hospital San Juan de Dios, 1995-1999

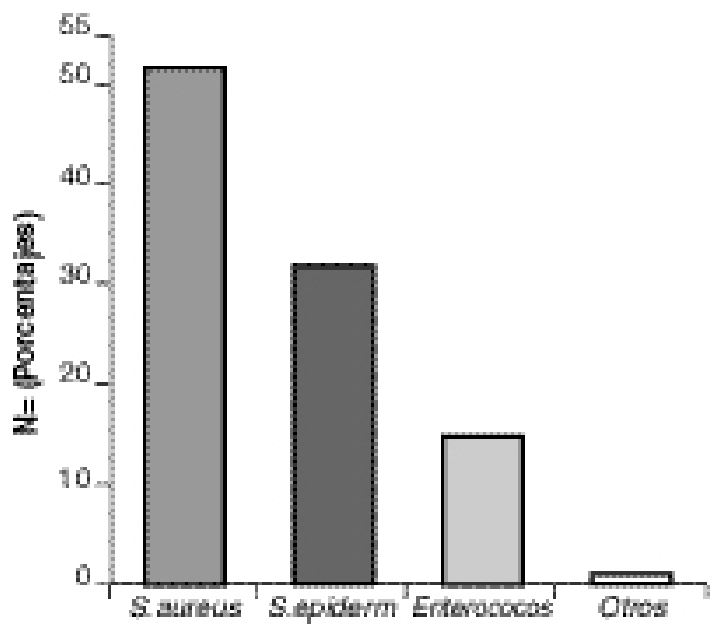

Figura 2

Resistencia a antibióticos en Bacilos Gram negativos Hospital San Juan de Dios, 1995-1999

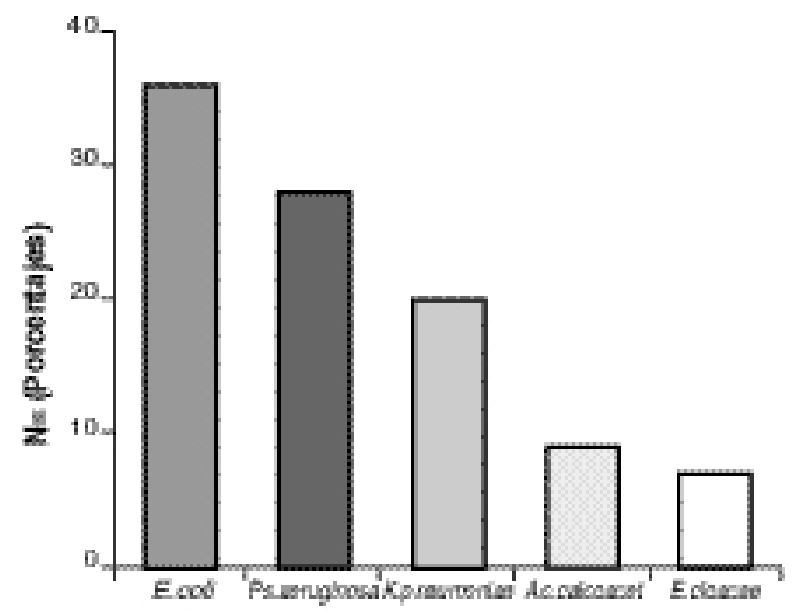

\begin{tabular}{|c|c|c|c|c|c|c|c|c|}
\hline \multicolumn{9}{|c|}{$\begin{array}{c}\text { Cuadro } 1 \\
\text { Porcentajes de sensibilidad Cocos Gram Positivos } \\
\text { Todos los sitios, Hospital San Juan de Dios, } \\
1995-1999\end{array}$} \\
\hline & \multicolumn{4}{|c|}{ Staphylococcus aureus* } & \multicolumn{4}{|c|}{ Staphylococcus epidermidis* } \\
\hline $\begin{array}{c}\text { Año } \\
\text { (aislamientos) }\end{array}$ & $\begin{array}{c}95 \\
(287)\end{array}$ & $\begin{array}{c}97 \\
(321)\end{array}$ & $\begin{array}{c}98 \\
(421)\end{array}$ & $\begin{array}{c}99 \\
(794)\end{array}$ & $\begin{array}{c}95 \\
(108)\end{array}$ & $\begin{array}{c}97 \\
(102)\end{array}$ & $\begin{array}{c}98 \\
(129)\end{array}$ & $\begin{array}{c}99 \\
(165)\end{array}$ \\
\hline Penicilina & 5 & 6 & 6 & 10 & 0 & 2 & 0 & 0 \\
\hline Cefalotina & 65 & 58 & 64 & 50 & 35 & 21 & 28 & 24 \\
\hline Oxacilina & 65 & 57 & 64 & 48 & 30 & 25 & 28 & 23 \\
\hline Clindamicina & 62 & 74 & 80 & 68 & 45 & 57 & 61 & 51 \\
\hline Vancomicina & 100 & 100 & 100 & 100 & 100 & 100 & 100 & 100 \\
\hline
\end{tabular}

Se detectó un aumento importante de la resistencia a la mayoría de los antibióticos por parte de A.calcoaceticus (Figura 3).

Es de anotar el incremento de la resistencia a ceftazidima principalmente en E.cloacae, K. pneumoniae y en A.calcoaceticus.

La resistencia de Escherichia coli, Klebsiella pneumoniae y Enterobacter cloacae a la ampicilina fue muy alta (64\%,97\% y 100\% respectivamente en el año 1999; Cuadro 3). Asimismo, llama la atención la alta resistencia de $K$. pneumoniae y E. cloacae tanto a la ampicilina como a 


\begin{tabular}{|c|c|c|c|c|c|c|c|c|c|c|c|c|}
\hline \multicolumn{13}{|c|}{$\begin{array}{c}\text { Cuadro } 2 \\
\text { Porcentajes de sensibilidad Enterobacterias. } \\
\text { Todos los sitios, Hospital San Juan de Dios } \\
1995-1999\end{array}$} \\
\hline \multirow{2}{*}{$\begin{array}{l}\text { Bacteria } \\
\text { Año } \\
\text { (aislamientos) }\end{array}$} & \multicolumn{4}{|c|}{ Escherichia coli } & \multicolumn{4}{|c|}{ Klebsiella pneum } & \multicolumn{4}{|c|}{ Enterobacter cloacae } \\
\hline & $\begin{array}{c}95 \\
(675)\end{array}$ & $\begin{array}{c}97 \\
(760)\end{array}$ & $\begin{array}{c}98 \\
(926)\end{array}$ & $\begin{array}{c}99 \\
(1073)\end{array}$ & $\begin{array}{c}95 \\
(367)\end{array}$ & $\begin{array}{c}97 \\
(428)\end{array}$ & $\begin{array}{c}98 \\
(335)\end{array}$ & $\begin{array}{c}99 \\
(372)\end{array}$ & $\begin{array}{c}95 \\
(153)\end{array}$ & $\begin{array}{c}97 \\
(175)\end{array}$ & $\begin{array}{c}98 \\
(167)\end{array}$ & $\begin{array}{c}99 \\
(218)\end{array}$ \\
\hline Amk & 100 & 93 & 85 & 85 & 80 & 74 & 67 & 68 & 90 & 83 & 100 & 75 \\
\hline Caz & 90 & * & 63 & 65 & 100 & * & 38 & 48 & 100 & * & 75 & 49 \\
\hline Ctx & 95 & 100 & 75 & 88 & 95 & 90 & 81 & 76 & 85 & 82 & 85 & 70 \\
\hline Cip & 90 & 88 & 83 & 82 & 80 & 90 & 83 & 85 & 75 & 90 & 85 & 83 \\
\hline Imip. & 100 & * & 100 & 100 & 100 & * & 100 & 100 & 100 & $*$ & 100 & 100 \\
\hline
\end{tabular}

\begin{tabular}{|c|c|c|c|c|c|c|c|c|c|c|c|c|}
\hline \multicolumn{13}{|c|}{$\begin{array}{c}\text { Cuadro } 3 \\
\text { Porcentajes de sensibilidad Enterobacterias. } \\
\text { Todos los sitios, Hospital San Juan de Dios } \\
1995-1999\end{array}$} \\
\hline \multirow{2}{*}{$\begin{array}{c}\text { Bacteria } \\
\text { Año } \\
\text { (aislamientos) }\end{array}$} & \multicolumn{4}{|c|}{ Escherichia coli } & \multicolumn{4}{|c|}{ Klebsiella pneum } & \multicolumn{4}{|c|}{ Enterobacter cloacae } \\
\hline & $\begin{array}{c}95 \\
(675)\end{array}$ & $\begin{array}{c}97 \\
(760)\end{array}$ & $\begin{array}{c}98 \\
(926)\end{array}$ & $\begin{array}{c}99 \\
(1073)\end{array}$ & $\begin{array}{c}95 \\
(367)\end{array}$ & $\begin{array}{c}97 \\
(428)\end{array}$ & $\begin{array}{c}98 \\
(335)\end{array}$ & $\begin{array}{c}99 \\
(372)\end{array}$ & $\begin{array}{c}95 \\
(153)\end{array}$ & $\begin{array}{c}97 \\
(175)\end{array}$ & $\begin{array}{c}98 \\
(167)\end{array}$ & $\begin{array}{c}99 \\
(218)\end{array}$ \\
\hline Amp & 38 & 42 & 35 & 38 & 10 & 2 & 3 & 3 & * & 0 & 6 & 0 \\
\hline Cefa & 67 & 54 & 50 & 57 & 44 & 52 & 50 & 48 & * & 9 & 9 & 6 \\
\hline Stx & 41 & 32 & 48 & 40 & 83 & 74 & 68 & 70 & 80 & 83 & 67 & 75 \\
\hline $\begin{array}{l}\text { *No hay datos } \\
\text { Amp, ampicilina }\end{array}$ & $c \epsilon$ & tina; & $x$ & tri & & & & & & & & \\
\hline
\end{tabular}

la cefalotina. Sin embargo, la resistencia a sulfa trimetoprin de estos gérmenes se mantuvo estable en el período analizado.

\section{Discusión}

La vigilancia epidemiológica constituye en un factor de gran importancia para el control de la resistencia bacteriana a antibióticos.

Analizaremos los datos obtenidos en este estudio según los grupos bacterianos y haremos una correlación con la experiencia obtenida en otras latitudes.

\section{Cocos Gram positivos}

1. Staphylococcus aureus: desde los años cincuenta se empezó a demostrar resistencia a la penicilina en esta bacteria. ${ }^{1,9}$ En esta serie, más del $90 \%$ de las cepas estudiadas presentaron este problema, relacionado directamente a la producción de $\beta$-lactamasa ( $>90 \%$ de las cepas eran productoras de esta enzima). Similares resultados se han encontrado en otros estudios. ${ }^{3,5-10}$ Desde los años setenta se ha observado un aumento de la resistencia a la meticilina y a la oxacilina, denominadas penicilinas resistentes a ß-lactamasas. ${ }^{9-12}$ El mecanismo responsable de este fenómeno es una alteración en las proteínas ligadoras de penicilina. ${ }^{9}$ En Estados Unidos y en Latinoamérica esta resistencia se encuentra presente aproximadamente en el $50 \%$ de las cepas estudiadas ${ }^{3,6}$ similar a lo demostrado en este estudio. Se han identificado varios factores de riesgo asociados a este fenómeno. ${ }^{9,11,12}$ La resistencia a la vancomicina se ha demostrado en algunas cepas aisladas en Japón; sin embargo, por el momento no pareciera constituir un problema médico, no obstante, sí se han identificado aislamientos de esta bacteria en diferentes partes del mundo, con sensibilidad disminuida a la vancomicina. ${ }^{11}$ En el presente estudio no se identificó ningún estafilococo resistente a la vancomicina. 
2. Staphylococcus epidermidis: Es ampliamente reconocida la alta resistencia a los diversos antibióticos por parte de estas bacteria., ${ }^{3,10,11}$ Este es un germen cuya incidencia en los diversos procesos infecciosos intrahospitalarios ha ido en aumento, fundamentalmente en pacientes internados en unidades de cuidado intensivo y que han requerido catéteres vasculares u otros métodos invasivos. ${ }^{3,5,11}$ En el Hospital San Juan de Dios, es el cuarto germen en cuanto a frecuencia, aislado de sangre (Boza R y Barrantes E, datos no publicados). Es de resaltar, sin embargo, que la sensibilidad a la clindamicina aún se mantiene cercana al $50 \%$ y que no se encontraron cepas resistentes a la vancomicina.

3. Enterococos: En los últimos años ha causado gran alarma en la comunidad médica mundial la aparición y rápida diseminación de enterococos resistentes no solo a la penicilina sino a cefalosporinas, aminoglucósidos y últimamente a la vancomicina. ${ }^{13-16}$ La producción de proteínas ligadoras de penicilina con baja afinidad así como la producción de ß-lactamasas y otras enzimas, son los mecanismos que explican la resistencia a los tres primeros grupos de antibióticos, mientras que la resistencia a vancomicina se debe a una alteración en las ligasas, enzimas involucradas en la unión D-ala-D-ala, paso inicial en la síntesis del peptidoglucano de la pared bacteriana de los CGP. ${ }^{13,14}$

Este mecanismo de resistencia se denomina VanA cuando el gen involucrado se encuentra en plásmidos, es decir, es transferible y es capaz de inducir altos grados de resistencia al antibiótico, con concentraciones inhibitorias mínimas del orden de $>64 \mathrm{ug} / \mathrm{ml} .{ }^{15}$ La mayoría de las epidemias de enterococos resistentes a vancomicina descritas hasta ahora son de este fenotipo y Enterococcus

\section{Figura 3 \\ Resistencia a antibióticos Acinetobacter calcoaceticus Hospital San Juan de Dios, 1995-1999}

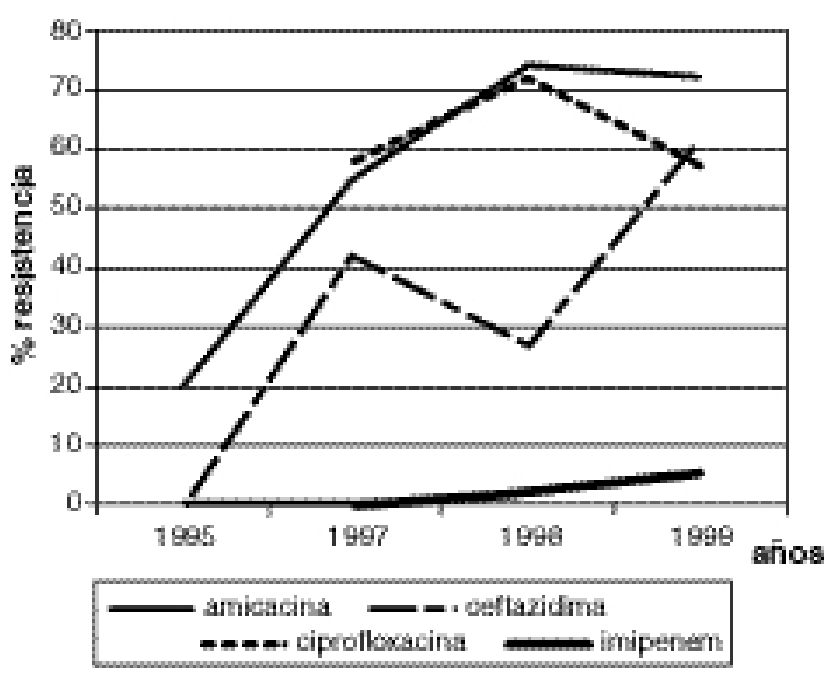

\section{Cuadro 4}

Porcentajes de sensibilidad Cocos Gram positivos Todos los sitios, Hospital San Juan de Dios, 1995-1999

\begin{tabular}{|c|c|c|c|c|c|c|c|c|}
\hline \multirow[b]{2}{*}{$\begin{array}{c}\text { Año } \\
\text { (aislamientos) }\end{array}$} & \multicolumn{4}{|c|}{ Ps. aeruginosa } & \multicolumn{4}{|c|}{ Acin. calcoaceticus } \\
\hline & $\begin{array}{c}95 \\
(420)\end{array}$ & $\begin{array}{c}97 \\
(447)\end{array}$ & $\begin{array}{c}98 \\
(609)\end{array}$ & $\begin{array}{c}99 \\
(686)\end{array}$ & $\begin{array}{c}95 \\
(156)\end{array}$ & $\begin{array}{c}97 \\
(178)\end{array}$ & $\begin{array}{c}98 \\
(233)\end{array}$ & $\begin{array}{c}99 \\
(103)\end{array}$ \\
\hline Amicacina & 85 & 78 & 70 & 77 & 80 & 45 & 26 & 28 \\
\hline Ceftazidma & 85 & 92 & 93 & 85 & 100 & 58 & 73 & 39 \\
\hline Ceftriaxona & 85 & * & 80 & $\star$ & 85 & 64 & 66 & 40 \\
\hline Ciprofloxacina & 80 & 75 & 70 & 63 & * & 42 & 28 & 43 \\
\hline Imipenem & 95 & 93 & 92 & 88 & 100 & 100 & 98 & 95 \\
\hline
\end{tabular}

faecium ha sido el principal involucrado. ${ }^{14}$ Sin embargo se ha demostrado la posibilidad de la transferencia de estos genes a Enterococcus faecalis, el más frecuentemente aislado en muestras clínicas, y a estafilococos. ${ }^{16}$ En nuestra serie, no se logró demostrar resistencia a la vancomicina en los enterococos aislados, lo que podría deberse a que la mayoría de las cepas identificadas fueron de E. faecalis, además de que el sistema VITEK ${ }^{\circledR}$ utilizado en el presente estudio es deficiente para detectar la resistencia a vancomicina en enterococos.

Se observó $20 \%$ de resistencia a la penicilina y en $50 \%$ de los aislamientos no se demostró sinergismo de la gentamicina con los antibióticos que alteran la síntesis de la pared bacteriana, lo que representa un grave problema ya que sabemos que las infecciones por estas bacterias no deben ser tratadas con un solo antibiótico ya sea un ß-lactámico, un glucopéptido o un aminoglucósido, sino que deben utilizarse combinaciones de estos grupos de antimicrobianos que son sinérgicos in vitro e in vivo, por lo que las opciones terapéuticas se disminuyen sensiblemente. ${ }^{1,2,13,14}$ Deben realizarse más estudios en nuestro hospital con el fin de aclarar estos aspectos del comportamiento de los enterococos.

4. Streptococcus pneumoniae: Este germen es causa frecuente de otitis media, sinusitis, bronquitis, neumonía y meningitis extrahospitalarias y septicemia. ${ }^{17}$ Clásicamente las penicilinas han sido altamente efectivas contra esta bacteria, no obstante en los últimos años se ha demostrado un aumento en la resistencia a estos antibióticos, de tal forma que en algunas regiones de Europa, Estados Unidos y Latinoamérica, de 7 a $60 \%$ de los neumococos aislados poseen resistencia alta o intermedia a la penicilina. ${ }^{6,7,17-20}$ Asimismo, se ha demostrado resistencia a otros antibióticos B-lactámicos, macrólidos y tetraciclinas en estos gérmenes. ${ }^{19}$ Se ha encontrado que la multirresistencia en Estados Unidos alcanza cifras entre 9-25\%. ${ }^{21}$ En las cepas analizadas en este estudio, todas provenientes de pacientes adultos, prácticamente no se encontró resistencia a la penicilina, dato que debe resaltarse ya que 
únicamente en estudios mexicanos ${ }^{22}$ encontramos cifras tan bajas de resistencia a la penicilina en los neumococos. Deberán realizarse estudios prospectivos con el fin de analizar esta situación.

5. Enterobacterias: Las enterobacterias poseen, al menos, tres mecanismos generales de resistencia a antimicrobianos, a saber, producción de enzimas inactivadoras de antibióticos (ß-lactamasas), alteración de los sitios blanco de los antibióticos (girasas de ADN en fluoroquinolonas) e impedir el acceso del antibiótico al sitio blanco bacteriano (impermeabilización de la membrana, bombas de eflujo). ${ }^{1-3}$ La producción de B-lactamasas es uno de los mecanismos más frecuentemente encontrados y estudiados en estas bacterias. Se han descrito varias de estas enzimas y su clasificación es compleja. ${ }^{23}$

En los últimos años han sido publicados reportes sobre varias epidemias en hospitales, con altas tasas de morbi-mortalidad, causadas por enterobacterias productoras de un tipo nuevo de B-lactamasas, las denominadas de espectro ampliado, las que les confieren resistencia a diversas penicilinas, cefalosporinas de $1^{\mathrm{a}}$ generación pero principalmente a cefalosporinas de $3^{\mathrm{a}}$ generación y aztreonam. ${ }^{24-33}$ Se desconoce la razón, pero estas bacterias presentan frecuentemente resistencia también a otros antibióticos como las fluoroquinolonas y los aminoglucósidos. ${ }^{25,26,31}$ Los genes se localizan en plásmidos por lo que fácilmente pueden transmitirse entre las bacterias. En $K$. pneumoniae y en menor grado $E$. coli y en otras enterobacterias, se ha identificado esta enzima. ${ }^{24,25,28} \mathrm{Se}$ ha establecido que un indicador de su presencia es el incremento de la resistencia a ceftazidima en K.pneumoniae, sin embargo existen métodos adecuados para su identificación completa. ${ }^{26}$ En el presente trabajo, la resistencia a ceftazidima en K.pneumoniae pasó de $0 \%$ en 1995 a $52 \%$ en 1999 y a cefotaxima de $5 \%$ a $24 \%$. Igualmente se demostró un aumento de la resistencia en E. coli. Aún cuando las ß-lactamasas de espectro ampliado confieren alta resistencia a las cefalosporinas de tercera generación, básicamente las oximino-cefalosporinas, esto es, a la ceftazidima, puede aparecer resistencia a cefotaxima, como se demostró en esta serie, de ahí la importancia de su reconocimiento. Se ha establecido que enterobacterias con CIM >2 ug/ml a la ceftazidima, cefotaxima, ceftriaxona o aztreonam deben considerarse como productoras de esta enzima y por lo tanto, debe investigarse su presencia. ${ }^{28-30}$ Las cefalosporinas de $3^{\mathrm{a}}$ generación, principalmente las oximino-cefalosporinas y también algunas metoximino-cefalosporinas como cefotaxima, no deben utilizarse si se demuestran bacterias productoras de esta enzima como causa del proceso infeccioso, principalmente en el caso de septicemias. ${ }^{31,32,34}$ Por otro lado, a pesar de que in vitro esta enzima es sensible a los inhibidores de B-lactamasa, únicamente para el tratamiento de infecciones urinarias se ha demostrado, hasta el momento, la utilidad de antibióticos B-lactámicos asociados a inhibidores de B-lactamasa. ${ }^{30-34}$ Según algunos autores ${ }^{32,34}$ los carbapenémicos asociados a aminoglucósidos son los antibióticos de primera elección en estos casos. Dado que en nuestro hospital K.pneumoniae es la bacteria más frecuentemente aislada de sangre y la segunda del tracto respiratorio y de la orina y el tercer BGN aislado en general (Boza R y Barrantes E, datos no publicados), estos hallazgos cobran gran relevancia, por lo que deben realizarse esfuerzos por analizar el comportamiento de estas enzimas en nuestra flora bacteriana.

La resistencia de K.pneumoniae a la ampicilina y a las cefalosporinas de $1^{\text {a }}$ generación puede explicarse tanto por la producción de $\beta$-lactamasas de espectro ampliado como ya fue descrito anteriormente, así como por otras ß-lactamasas no inducibles del grupo $1 . .^{23,28}$

Se demostró un aumento considerable en la resistencia a aminoglucósidos y en menor medida a las quinolonas en el período estudiado. Este fenómeno se ha demostrado que está relacionado con el uso indiscriminado de estos medicamentos y como se explicaba anteriormente, con el incremento de la producción de $\beta$-lactamasas de espectro ampliado. ${ }^{28,29,31}$ Los principales mecanismos de resistencia encontrados en las enterobacterias a esos medicamentos son la producción de enzimas modificadoras de aminoglucósidos, la impermeabilización de la membrana bacteriana al ingreso de estos antibióticos y la presencia de mecanismos de eflujo y alteraciones en la ADN-girasa blanco. ${ }^{28,34}$

6. Enterobacter cloacae: Esta bacteria correspondió al 7\% de los BGN aislados, no obstante en algunos países ha aumentado la frecuencia de su identificación en diversos procesos infecciosos así como se ha observado un aumento de la resistencia a antibióticos en esta bacteria por lo que se analiza por aparte. ${ }^{26,29}$ En este estudio se demostró una alta resistencia a las cefalosporinas de primera generación (>90\%) y a la ampicilina (>95\%), con un incremento de $25 \%$ en 1998 a $51 \%$ en 1999 en la resistencia a ceftazidima y de $15 \%$ en 1995 a $30 \%$ en 1999 a cefotaxima. La resistencia a antibióticos ß-lactámicos en estas bacterias es mediada por la ß-lactamasa AmpC cuyo gene se localiza en los cromosomas y es fácilmente inducida su expresión por el uso de cefalosporinas de amplio espectro como las de $3^{\mathrm{a}}$ generación. ${ }^{28,29}$ Por presión selectiva al utilizar estas cefalosporinas, las cepas bacterianas mutantes que persisten son altamente productoras de ß-lactamasa que actúan sobre todos los antibióticos ß-lactámicos conocidos excepto los carbapenémicos. Es decir el uso amplio de cefalosporinas de tercera generación conlleva la aparición de resistencia en esta bacteria. Podríamos suponer que esta ß-lactamasa es altamente activa contra la ampicilina y las cefalosporinas de primera generación y a las mismas concentraciones menos activa contra cefalosporinas de tercera generación, pero al entrar en contacto con éstas se producen grandes cantidades de la enzima, lo que explicaría el comportamiento de la sensibilidad a los antibióticos ß- 
lactámicos demostrados en este estudio. Por otro lado, este fenómeno ha sido identificado en E. cloacae y en menor proporción en $E$. coli $^{28,29,31,32}$ lo que explicaría los hallazgos en este trabajo.

7. BGN no fermentadores: (Pseudomonas aeruginosa y Acinetobacter calcoaceticus): en Norteamérica en general, Pseudomonas aeruginosa es el principal germen aislado de este grupo. ${ }^{35}$ Sin embargo, en Latinoamérica y en algunas regiones de los Estados Unidos, Acinetobacter calcoaceticus se ha constituido en una bacteria muy importante tanto por su creciente identificación como germen intrahospitalario como por su alta tasa de resistencia a antibióticos. ${ }^{6,7,36-38}$ Ps.aeruginosa fue el segundo BGN aislado en frecuencia en este estudio, así como el tercero en importancia aislado del tracto respiratorio y de orina en el hospital San Juan de Dios (Boza R y Barrantes E; datos no publicados). En Ps. aeruginosa se observó un aumento de 15 a $23 \%$ en la resistencia a amicacina, una sensibilidad estable hacia la ceftazidima, pero un aumento moderado de la resistencia a imipenem e importante a la ciprofloxacina. En A. calcoaceticus el panorama es alarmante, ya que la resistencia a todos los antibióticos con excepción de imipenem, es muy alta. Hallazgos similares han sido demostrados en otras regiones, ${ }^{3,5-8,37,38}$

No obstante, preocupa el hecho de encontrar cada vez con mayor frecuencia notificaciones en la literatura mundial sobre el incremento de la resistencia al imipenem en este grupo bacteriano. ${ }^{35-38}$ La resistencia a los antibióticos ß-lactámicos en estas bacterias es mediada por la producción de $\beta$-lactamasas constitutivas o inducibles ${ }^{1,2,35,36}$ y menos frecuentemente, adquiridas ${ }^{36}$ así como por la impermeabilización de la membrana al ingreso de estos medicamentos. ${ }^{28,35}$ En cuanto a los aminoglucósidos, los mecanismos de resistencia son similares a los encontrados en otros BGN. La resistencia a las quinolonas (ciprofloxacina), está dada tanto por mutaciones en la ADN-girasa como por incremento en los mecanismos de eflujo, ambos mecanismos dependientes de genes cromosómicos. ${ }^{31,35}$

En conclusión, se demostró en este estudio alta resistencia en los CGP a la penicilina y a la oxacilina, con una sensibilidad estable a la clindamicina y no se demostró resistencia a la vancomicina en ninguna de estas bacterias. Solo el $50 \%$ de los enterococos presentaron sinergismo de la gentamicina in vitro con los antibióticos que actúan sobre la pared bacteriana.

En los BGN, se encontró una resistencia alta a los antibióticos B-lactámicos, con excepción del imipenem. En los BGN no fermentadores se observó una alta incidencia de multirresistencia, tanto a antibióticos B-lactámicos y aminoglucósidos como a las quinolonas, pero una sensibilidad estable a imipenem, similar a lo publicado en otras regiones.

Estos resultados exigen tomar medidas con el fin de enfrentar este problema, las que deben incluir el focalizar los servicios de alto riesgo (UCI, cirugía entre otros), desarrollar pro- gramas de educación, incrementar las medidas higiénicas en el hospital, reforzar los protocolos para el uso de antibióticos de uso restringido por especialistas y valorar el "reciclaje" de medicamentos antibacterianos. $3,9,12,15,20,27,30,38,39$

\section{Abstract}

Background and aim: Antimicrobial resistance results in increased morbidity, mortality and costs of health care. Therefore, surveillance of antimicrobial resistance in hospitals must be part of infection control programs.

The Hospital San Juan de Dios is a tertiary health care center in Costa Rica with 700 beds for adults. Since 1995, an automatized system (VITEK $®$ ) is used in our bacteriology laboratory allowing an adequate control of the resistance patterns of bacterias isolated from different sites.

The aim of this report is to describe the susceptibility to some antibiotics found in bacterias isolated from different sites from 1995 to 1999 and to determine their changes in resistance.

Materials and Methods: Were reviewed. The files of the bacteriology laboratory of the hospital was done. We analized bacterial strains from 1995 to 1999. Data from 1996 were incomplete and were not studied. All isolates were analized in the automatized system VITEK ${ }^{\circledR}$. Streptococcus pneumoniae strains from blood or sputum were studied by the E-test method and their sensitivity ranges met the NCCLS criteria.

Results: We studied 2817 Gram positive cocci (GPC) strains and 7626 isolates of Gram negative bacteria (GNB). Staphylococcus aureus was the most frequently GPC specie found and Escherichia coli was the most common GNB.

More than $90 \%$ of staphylococcal species produced B-lactamase and were resistant to penicillin.

Resistance to oxacillin (methicillin) in Staphylococcus aureus increased from $35 \%$ to $52 \%$ in this period. In coagulase negative staphylococci the resistance to this antibiotic was very high $(70-77 \%)$.

Susceptibility to cephalotin and clindamycin in Staphylococcus aureus was similarly high (50-60\%) in the years studied and in coagulase negative staphylococci (Staphylococcus epidermidis) the resistance stayed in high levels (65-70\%). There were not resistance to vancomycin in the staphylococci analyzed.

Ninety eight per cent of the 61 isolates of Streptococcus pneumoniae were susceptible to penicillin.

Susceptibility to penicillin was observed in $80 \%$ of 483 isolates of Enterococcus faecalis. Fifty percent of the enterococci showed synergism between gentamicin and cell-wall active agents.

Only a few isolates of Enterococcus faecuim were analized and no resistance to vancomycin was found. Resistance to 
aminoglycosides was demonstrated in $50 \%$ of enterococci isolates.

Regarding enterobacterias, Escherichia coli strains presented high resistance to ampicillin (60\%), sulfa trimetoprin (60\%) and a decrease in the susceptibility to ceftazidime from $90 \%$ in 1995 to $65 \%$ in 1999 , to cefotaxime from $95 \%$ to $88 \%$, from $67 \%$ to $57 \%$ to cephalotin and from $100 \%$ to $85 \%$ to amikacin in the same period. Resistance to ciprofloxacin was $10 \%$ in 1995 and $20 \%$ in 1999.

Regarding Klebsiella pneumoniae, resistance to amikacin increase from $20 \%$ to $32 \%$, to ceftazidime from $0 \%$ to $52 \%$, to cefotaxime from $5 \%$ to $24 \%$ but susceptibility to ciprofloxacin was high in the period (80-90\%). Resistance to ampicillin was present in more than $90 \%$ of the K.pneumoniae isolates and to cephalotin in more than $50 \%$ of this bacteria.

Enterobacter cloacae showed high resistance patterns to ampicillin (>95\%), cephalotin (> 90\%) and ceftazidime (25$50 \%)$.

In non-fermenting Gram negative bacilli, resistance to amikacin $(20 \%-72 \%)$ ceftazidime $(42 \%-61 \%)$ and ciprofloxa$\operatorname{cin}(58 \%)$ was documented in Acinetobacter calcoaceticus. In Pseudomonas aeruginosa an increase in the resistance to amikacin (15\%-30\%) and ciprofloxacin(20\%-37\%) were noted.

In all BGN strains studied, a high susceptibility pattern to imipenem (from $88 \%$ in Ps. aeruginosa and $95 \%$ in Ac. calcoaceticus to $100 \%$ in enterobacterias) were observed.

Discussion: We analized these findings and compared them with those found in the medical literature. High resistance patterns to penicillin and oxacillin in staphylococci strains were documented. High resistance in GNB to ampicillin, cephalotin, ceftazidime and ciprofloxacin was observed. High susceptibility patterns to imipenem in GNB were found.

Key words: antibiotics, antibiotic resistance, bacterial infections, beta-lactam resistance, vancomycin resistance, pneumococcus drug resistance, broad spectrum $\beta$-lactamases.

\section{Referencias}

1. Opal SM, Mayer KH, Medeiros A. Mechanisms of Bacterial Antibiotic Resistance. In: Mandell GL Douglas JE and Dolin R eds. Mandell Douglas and Bennett's. Principles and Practice of Infectious Diseases New York Churchill-Livingstone Fifth Edition 2000; 236-252.

2. Tavares W. Resistencia Bacteriana. En: Tavares W Manual de Antibióticos e Quimioterápicos Antiinfecciosos São Paulo Atheneu Secunda Edicão 1996; 43-100.

3. Shlaes DM, Gerding DN, John JF, Craig WA, Borstein DL, Duncan RA, et al. Society for Healthcare Epidemiology of America and Infectious Diseases Society of America Joint Committee on the Prevention of Antimicrobial Resistance: Guidelines for the Prevention of Antimicrobial Resistance in Hospitals. Clin Infect Dis 1997; 25:584-599.

4. Baquero F, Negri MC, Morosini MI, Blázquez J. Antibiotic-Selective Environmets. Clin Infect Dis 1998; 27 (Supp11): S5-11.
5. Fluit AdC, Jones ME, Schmitz FJ, Acar J, Gupta R, Verhoef J, et al. Antimicrobial Susceptibility and Frequency of Ocurrence of Clinical Blood Isolates in Europe from SENTRY Antimicrobial Surveillance Program,1997-1998. Clin Infect Dis 2000; 30: 454-460.

6. Sader HS, Jones RN, Pfaller MA, Latin America SENTRY Participant Group. An Assessment of Pathogen Frequency and Resistance Patterns in Selected Latin America Hospitals. Abstracts of the $39^{\text {th }}$ ICAAC September 1999; 177.

7. Bantar C, Famiglietti A, Goldberg M, Subcomisión de Antimicrobianos. A 3-Year Survey of Bacterial Resistance in Argentina from a National Program. Abstracts of the 39th ICAAC 1999; 175.

8. Ho M, Yeh LC, McDonald TL. The Taiwan Surveillance of Antimicrobial Resistance System. Abstracts of the $39^{\text {th }}$ ICAAC September 1999; 183.

9. Peters G, Becker K. Epidemiology, control and treatment of methici1lin-resistant Staphylococcus aureus. Drugs 1996; 52(Suppl2): 50-54.

10. Kreiswirth B, Kornblum J, Arbeit RD. Evidence for clonal origin of methicillin resistance in Staphylococcus aureus. Science 1993; 259: 227-230.

11. Tenover FC, Lancaster MV, Hill BC. Characterization of staphylococci with reduced susceptibilities to vancomycin and other glycopeptides. J Clin Microbiol 1998; 36: 1020-1027.

12. Landman D, Chockalingam M, Quale JM. Reduction in the incidence of methicillin-resistant Staphylococcus aureus and ceftazidime-resistant Klebsiella pneumoniae following changes in a hospital antibiotic formulary. Clin Infect Dis 1999; 28: 1062-1066.

13. French GL. Enterococci and Vancomycin Resistance. Clin Infect Dis 1998; (Suppl1): S75-83.

14. Reynolds PE. Glycopeptide resistance in Gram positive bacteria. J Med Microbiol 1992; 36:14-17.

15. Leclercq R, Courvalin P. Resistance to glycopeptides in enterococci. Clin Infect Dis 1997; 24: 545-556.

16. Gambarotto K. Prevalence of Vancomycin-Resistant Enterococci in Fecal Samples from Hospitalized Patients and Nonhospitalized Controls in a Cattle-Rearing Area of France. J Clin Microbiol 2000; 38: 620-624.

17. Pallares R, Gudiol R, Linares J, Vadillo M, Alonso T. Risk factors and response to antibiotic therapy in adults with bacteremic pneumonia caused by penicillin-resistant pneumococci. N Engl J Med 1987; 317 : $18-22$.

18. Marton A. Pneumococcal antimicrobial resistance: the problem in Hungary. Clin Infect Dis 1992; 15: 106-111.

19. Friedland IR, McCracken JR. Management of infections caused by antibiotic resistant Streptococcus pneumoniae. N Engl J Med 1994; 331: 377-382.

20. Reinert RR, Simic S, Al-Lahham A, National Surveillance Study Group. Antimicrobial Resistance of Streptococcus pneumoniae from Outpatients with Respiratory Tract Infections in Germany from 1998 to 1999: Results of a National Surveillance Study. J Clin Microbiol 2001; 39: 1187-1189.

21. Doern GV, Pfaller MA, Kugler K, Sentry Antimicrobial Surveillance Program. Prevalence of antimicrobial resistance among respiratory tract isolates of Streptococcus pneumoniae in North America: 1997 results from the SENTRY antimicrobial surveillance program. Clin Infect Dis 1998; 27:764-770. 
22. Sifuentes-Osornio J, Donís-Hernández J, Arredondo-García JL, Escalante Ramírez O, Macías A, Muñoz JM, et al. Informe sobre resistencia bacteriana: Estudio piloto en seis centros de México. Rev Panamer Infect 1999; 3 (supl1): S45-S47.

23. Bush K, Jacoby GA, Medeiros AA. A functional classification scheme for B-lactamase and its correlation with molecular structure Antimicrob Agents Chemother 1995; 39: 1211:1233.

24. Jarlier V, Nicolas MH, Fournier G, Phillipon A. Extended broad-spectrum $\beta$ - lactamases conferring transferable resistance to newer B-lactam agents in Enterobacteriaceae: hospital prevalence and susceptibility patterns. Rev Infect Dis 1988; 10: 867-878.

25. Papanicolau GA, Medeiros AA, Jacoby GA. Novel plasmid-mediated B-lactamase conferring resistance to oxyimino and methoxy B-lactams in clinical isolates of Klebsiella pneumoniae. Antimicrob Agents Chemother 1990; 34: 2200-2209.

26. Fekete T, Tumah H, Woodwell J, Satishchandran V, Truant A, Axelrod P. Comparative Susceptibilities of Klebsiella Species, Enterobacter Species and Pseudomonas aeruginosa to 11 Antimicrobial Agents in a Tertiary-Care University. Am J Med 1996; 100 (suppl 6A): 20S-25S.

27. Lautenbach E, Patel JB, Bilker WB. Extended-Spectrum B-LactamaseProducing Escherichia coli and Klebsiella pneumoniae: Risk Factors for Infection and Impact of Resistance on Outcomes. Clin Infect Dis 2001; 32: 1162-1171.

28. Pitout JDD, Sanders CC, Sanders E. Antimicrobial Resistance with Focus on B-Lactam Resistance in Gram-negative Bacilli. Am J Med 1997; 103: 51-59.

29. Saurina G, Quale JM, Manikal VM, Oydna E, Landman D. Antimicrobial resistance in Enterobacteriaceae in Brooklyn, NY: epidemiology and relation to antibiotic usage patterns. J Antimicrob Chemother 2000; 45: 895-898.

30. Pena C, Pujol M, Ardanuy C, Ricart A, Pallares R, Linares J, et al. Epidemiology and successful control of a large outbreak due to Klebsiella pneumoniae producing extended-spectrum beta-lactamases. Antimicrob Agents Chemother 1998; 42: 53-58.
31. Paterson DL, Mulazimoglu L, Casellas JM, Ko WC, Goossens H, Von Gottberg A, et al. Epidemiology of ciprofloxacin resistance and its relationship to extended-spectrum beta-lactamase production in Klebsiella pneumoniae isolates causing bacteremias. Clin Infect Dis 2000; 30: 473-478.

32. Paterson DL, Bolmstrom A, Karlsson A, Goransson E Activity of Antibiotic Combinations against Extended -Spectrum Beta-Lactamase (ESBL)-Producing Klebsiella pneumoniae. Abstracts of the $39^{\text {th }}$ ICAAC September 1999; 291.

33. Gales AC, Bolmstrom A, Sampaio J, Jones RN, Sader HS. Antimicrobial Susceptibility of Klebsiella pneumoniae Producing Extended-Spectrum beta-lactamase (ESBL) Isolated in Hospitals in Brazil. Braz J Infect Dis 1997; 1: 196-203.

34. Paterson DL Infection Control and Therapeutic Options for Extended Spectrum -Lactamases Producers. Abstracts of the $40^{\text {th }}$ ICAAC Toronto 2000 .

35. Hancock REW, Resistance Mechanisms in Pseudomonas aeruginosa and Other Non- Fermentative Gram Negative Bacteria. Clin Infect Dis 1998; 27(Suppl1): S93-S99.

36. Cornaglia G, Mazzariol A, Laureti L, Rossolini GM, Fontana R. Hospital Outbreak of Carbapenem-Resistant Pseudomonas aeruginosa Producing VIM-1, a Novel Transferable Metallo-\{-Lactamase. Clin Infect Dis 2000; 31: 1119-1125.

37. Villers D, Espaze E, Coste-Burel A. Nosocomial Acinetobacter baumanii infections: microbiological and clinical epidemiology. Ann Intern Med 1998; 129: 182-189.

38. Manikal VM, Landman D, Saurina G, Quale JM. Endemic Carbapenem-Resistant Acinetobacter Species in Brooklyn New York: Citywide Prevalence, Interinstitutional Spread, and Relation to Antibiotic Usage. Clin Infect Dis 2000; 31; 101-106.

39. Murthy R. Implementation of strategies to control antimicrobial resistance. Chest 2001; 119 (Suppl 2): 405S-411S. 